\title{
Soins médicaux intégrés dans le Val Müstair - la solution pour la périphérie?
}

Karin Hänni ${ }^{a}$,

Theodor von Fellenberg ${ }^{b}$

a Directrice/CEO Center da sandà Val Müstair

b Médecin-chef, Center da sandà Val Müstair

Le rapport mentionné sur les modèles de soins peut être consulté sur le site web de la CDS sous le thème Structures ambulatoires.
Correspondance:

Dr sc. nat. Karin Hänni

haenni[at]ovmgr.ch
Différentes solutions sont demandées pour garantir la médecine de premier recours. Dans la première partie de la présente série d'articles, le modèle de cabinet de groupe de médecins de campagne décentralisé et en réseau qui est en phase de réalisation dans le canton de Thurgovie a été décrit. En vue de susciter l'inspiration et la discussion, un modèle de soins intégrés cité à titre d'exemple dans le rapport de la Conférence des directeurs de la santé (CDS) et de l'Office fédéral de la santé publique (OFSP) sur les nouveaux modèles de soins pour la médecine de premier recours * est présenté ci-après.

\section{Tout sous le même toit}

Le centre de santé Center da sandà Val Müstair réunit sous le même toit le plus petit hôpital de Suisse et un cabinet intégré avec service de garde, mais aussi service de sauvetage, EMS, aide et soins à domicile, solvent toutefois pas le problème de la périphérie, parce que celui-ci tient en premier lieu à ce que 1 . les conditions de travail ne sont plus attractives (heures de piquet), 2. les formations nécessaires de médecin de premier recours ne sont plus offertes et 3 . le champ d'activité et l'évolution professionnelle sont restreints par la limitation des mandats de prestations.

Les soins intégrés sont par conséquent un concept qui influence positivement les processus de traitement, sont intéressants du point de vue économique et peuvent apporter des réponses à l'évolution démographique. Mais ils ne sont pas en mesure d'enrayer le problème principal: le risque de pénurie de personnel dans la périphérie.

Ce problème s'est accentué en 2003 dans le Val Müstair par la limitation du mandat de prestations à

\section{Il faut faire preuve d'innovation dans le recrutement du personnel en} offrant des conditions d'engagement attractives telles que le partage du travail, l'annualisation du temps de travail, l'embauche en même temps du ou de la partenaire.

dentiste, consultations de psychothérapie, physiothérapie, conseil parental, podologie et kinésiologie; il prend soin des 1700 habitants et des vacanciers. Ces prestations sont fournies par 90 collaborateurs et collaboratrices (45 équivalents plein temps), dont 3 médecins (médecin-chef, chef de clinique, médecin-assistant) et un poste de stage. Nombre de personnes remplissent plusieurs fonctions c'est-à-dire qu'elles peuvent intervenir dans différents domaines. Au sein de petites équipes «familiales» sans grande fluctuation du personnel, la collaboration interprofessionnelle va de soi.

Les soins intégrés sont financièrement attractifs parce que les interfaces n'engendrent pas de pertes par manque d'information ou doublons. Ils ne ré- la médecine interne. La conséquence en est que les soins d'urgence deviennent un véritable défi pour tout le personnel de par la diminution des cas de chirurgie, gynécologie et obstétrique générales. Cette baisse du nombre de cas signifie de plus que la charge de travail des collaborateurs et collaboratrices décroît sans que les effectifs puissent être réduits, parce que la qualité corrélative des structures est exigée.

\section{La clé du problème}

A notre avis, il faudrait suivre deux grands axes: 1 . le masterplan «Médecine de famille et médecine de base» du Conseil fédéral devrait avancer avec vigueur de sorte que des médecins de premier recours soient

\section{Série d'articles sur les nouveaux modèles pour le cabinet de médecine de famille} dans «PrimaryCare»

Depuis le mois de février, la revue PrimaryCare propose une série d'articles originaux et intéressants sur des modèles efficaces de cabinets médicaux. Vous les découvrirez sous www.primary-care.ch $\rightarrow$ Pour les lecteurs $\rightarrow$ Topic collections $\rightarrow$ Unternehmen Hausarztpraxis. 
formés pour les soins ambulatoires et stationnaires dans la périphérie. Ils doivent trouver dans la périphérie des alternatives professionnellement attractives aux disciplines spécialisées pratiquées dans les centres. 2. Dans l'intervalle, les cantons doivent selon nous examiner si la limitation des mandats de prestations est la bonne solution ou s'ils admettraient éventuellement la compensation des manques dans la qualité des structures dont disposent les prestataires en périphérie via des modèles innovants avec des partenaires (fonctions de coaching, coopérations, rotations). Nous développons dans le centre de santé des modèles qui anticipent l'évolution proposée. Jusqu'à ce que ces mesures déploient leurs effets, ne reste pour nous que la possibilité de faire preuve d'innovation dans le recrutement du personnel en offrant des conditions d'engagement attractive telles que le partage du travail, l'annualisation du temps de travail, l'embauche en même temps du ou de la partenaire.
Les systèmes de financement doivent en outre également être efficaces à long terme: le financement moniste pourrait atténuer l'énorme charge administrative - pas seulement en médecine mais pour tous les partenaires impliqués dans le processus de traitement et de financement.

A ces conditions, les soins intégrés sont un modèle de succès.

Aimeriez-vous présenter dans le cadre de notre
série d'articles une autre initiative que vous
connaissez visant à garantir et promouvoir la
médecine de famille?
Correspondance: Sibylle Christen, Conférence des
directeurs de la santé CDS, Maison des cantons,
Speichergasse 6 , Case postale 684,3000 Berne 7 ,
Tél. 0313562020 , Fax 0313562030 ,
sibylle.christen[at]gdk-cds.ch

Aimeriez-vous présenter dans le cadre de notre série d'articles une autre initiative que vous mouvoir la médecine de famille?

Correspondance: Sibylle Christen, Conférence des directeurs de la santé CDS, Maison des cantons, Tél. $031356 \quad 20 \quad 2$ sibylle.christen[at]gdk-cds.ch

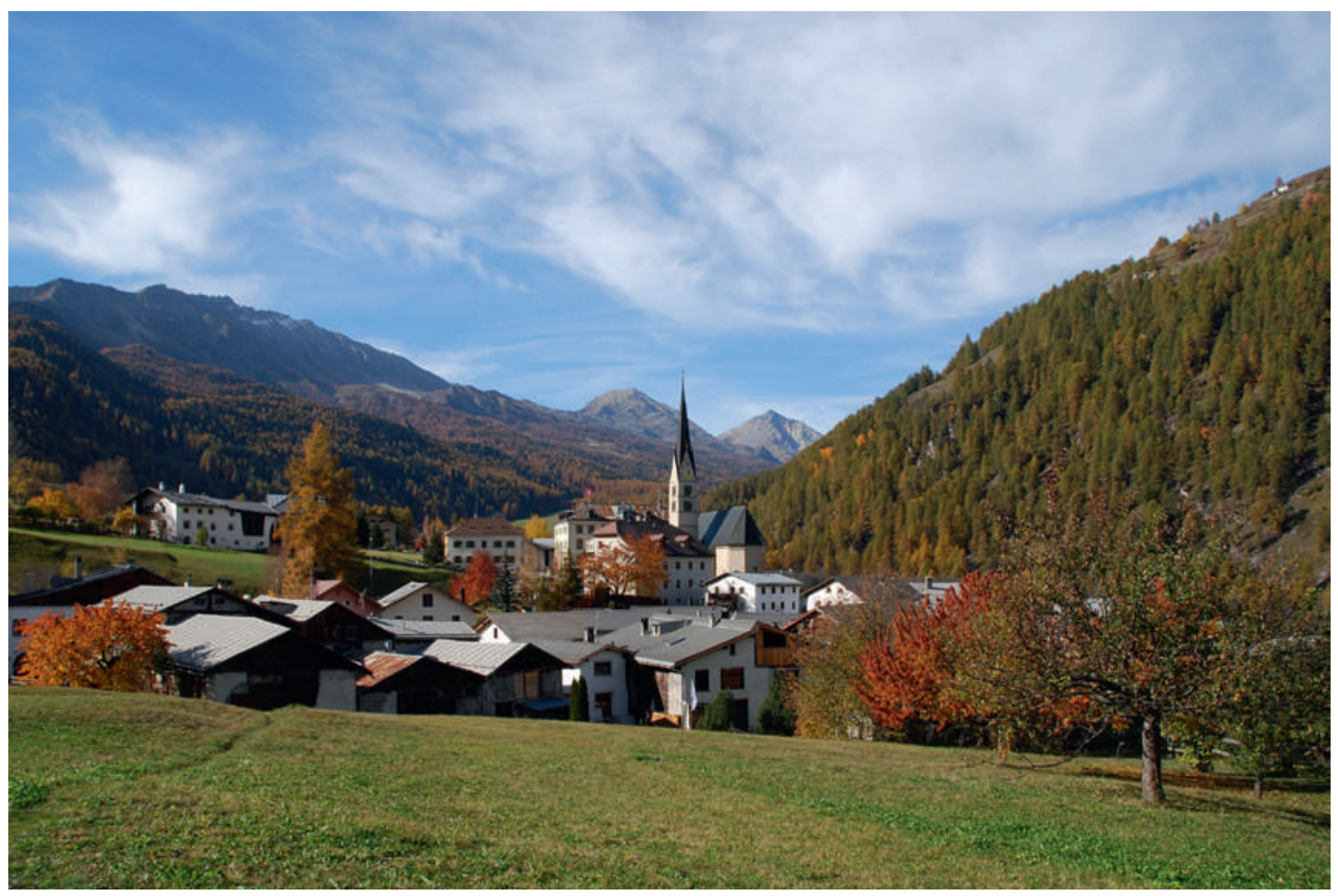

Le plus petit hôpital de Suisse se trouve au Val Müstair. II prend soin des 1700 habitants et des vacanciers.

๑) Wladyslaw. Utilisation conforme à la licence «art libre, www.artlibre.org 